\title{
Molecular Basis of Hepatic Carnitine Palmitoyltransferase I Deficiency
}

\author{
Lodewijk IJlst, ${ }^{*}$ Hanna Mandel, ${ }^{\S}$ Wendy Oostheim, ${ }^{*}$ Jos P.N. Ruiter, ${ }^{\star}$ Alisa Gutman,, and Ronald J.A. Wanders ${ }^{\star} \neq$ \\ $*$ Department of Clinical Chemistry and ${ }^{\ddagger}$ Department of Pediatrics, University of Amsterdam, Academic Medical Center, 1100 DE \\ Amsterdam, The Netherlands; ${ }^{\S}$ Department of Pediatrics, Rambam Medical Center, Haifa 31096, Israel; and $\|^{\|}$epartment of Clinical \\ Biochemistry, Hadassah University Hospital, Jerusalem 91120, Israel
}

\begin{abstract}
Mitochondrial fatty acid $\beta$-oxidation is important for energy production, which is stressed by the different defects found in this pathway. Most of the enzyme deficiencies causing these defects are well characterized at both the protein and genomic levels. One exception is carnitine palmitoyltransferase I (CPT I) deficiency, of which until now no mutations have been reported although the defect is enzymatically well characterized. CPT I is the key enzyme in the carnitine-dependent transport across the mitochondrial inner membrane and its deficiency results in a decreased rate of fatty acid $\beta$-oxidation. Here we report the first delineation of the molecular basis of hepatic CPT I deficiency in a new case. cDNA analysis revealed that this patient was homozygous for a missense mutation (D454G). The effect of the identified mutation was investigated by heterologous expression in yeast. The expressed mutant CPT IA displayed only $2 \%$ of the activity of the expressed wild-type CPT IA, indicating that the $\mathrm{D} 454 \mathrm{G}$ mutation is the disease-causing mutation. Furthermore, in patient's fibroblasts the CPT IA protein was markedly reduced on immunoblot, suggesting that the mutation renders the protein unstable. (J. Clin. Invest. 1998. 102:527-531.) Key words: carnitine palmitoyltransferase - fatty acid - Mendelian disorder $\bullet$ hereditary disease $\bullet$ gene expression
\end{abstract}

\section{Introduction}

The mitochondrial $\beta$-oxidation of long-chain fatty acids is a major source of energy, especially in the heart. Before their catabolism, long-chain fatty acids are activated to their CoA ester in the extramitochondrial space by long-chain acyl-CoA synthetases localized in the mitochondrial outer membrane as well as the peroxisomal and endoplasmic reticulum membrane. Transport of long-chain acyl-CoA esters across the mitochondrial inner membrane requires carnitine and three proteins including carnitine palmitoyltransferase I (CPT I), ${ }^{1}$ the carnitine acylcarnitine carrier (CAC), and CPT II. The first en-

Address correspondence to Prof. Dr. Ronald J.A. Wanders, Department of Pediatrics, Academic Medical Center, Meibergdreef 9 (Room F0-226), Amsterdam, 1105 AZ The Netherlands. Phone: 3120-5664197; FAX: 31-20-6962596; E-mail: wanders@AMC.UVA.NL

Received for publication 28 January 1998 and accepted in revised form 1 June 1998.

J. Clin. Invest.

(C) The American Society for Clinical Investigation, Inc. 0021-9738/98/08/0527/05 \$2.00

Volume 102, Number 3, August 1998, 527-531

http://www.jci.org zyme (CPT I) is localized in the outer-mitochondrial membrane and converts the fatty acyl-CoA ester to its carnitine ester. This carnitine ester is subsequently transported across the mitochondrial inner membrane by $\mathrm{CAC}$ which belongs to the mitochondrial carrier family. Once inside the mitochondrial lumen, CPT II reconverts the carnitine ester to the CoA ester which can then serve as a substrate for $\beta$-oxidation. CPT II is bound to the mitochondrial inner membrane facing the mitochondrial matrix. The relationship between CPT I and CPT II has long remained an enigma. However, recent studies notably by McGarry and co-workers have clearly established that CPT I and CPT II are distinct enzyme proteins encoded by different genes (for review see reference 1 ). There is only a single form of CPT II expressed in all tissues. On the other hand, two different isoforms of CPT I have been described with distinct tissue distributions. These two forms are called the hepatic (CPT IA) and muscle form (CPT IB) and are encoded by different genes localized on chromosome 11q13.1-13.5 and 22q13.3113.32, respectively (2).

Clinically, three different phenotypes can be distinguished as characterized by muscular (3), hepatic (4), or hepatocardiomuscular symptoms, respectively (5). In the hepatic type, CPT I is deficient, whereas CPT II is normally active. In the other types of CPT deficiency, CPT II is deficient to different extents with normal CPT I activity. After the first report on CPT I deficiency in 1981 (4) at least 11 cases with CPT I deficiency have been described (for overview see reference 6) presenting with coma, seizures, hepatomegaly, and hypoketotic hypoglycemia, usually after a period of fasting or after a viral infection. When appropriately treated, patients suffering from hepatic CPT I deficiency may survive normally. Remarkably, patients suffering from CPT I deficiency do not display chronic muscle weakness and/or cardiomyopathy, which is in marked contrast to other inherited deficiencies in the oxidation of long-chain fatty acids including CPT II deficiency.

The molecular basis of CPT I deficiency has so far remained unresolved although the cloning of human CPT IA was reported in 1995 (7). We now report the resolution of the molecular defect in a patient with enzymatically proven CPT I deficiency.

\section{Methods}

Case. Patient S.I. is the second child of consanguineous parents (2nd-3rd degree cousins). She presented at 15 mo of age with diarrhea and feeding difficulties. On admission she was severely hypotonic and lethargic. Physical examination revealed hepatomegaly, normal heart sounds and rhythm, and decreased tendon reflexes.

At time of admission she suffered from hypoketotic hypoglycemia (glucose $1.6 \mathrm{mmol} /$ liter). Further laboratory findings in her blood were (normal values between parentheses): $\mathrm{pH} 7.2, \mathrm{HCO}_{3} 11 \mathrm{mmol} /$

1. Abbreviations used in this paper: CAC, carnitine acylcarnitine carrier; CPT, carnitine palmitoyltransferase. 
liter, base excess $12 \mathrm{mmol} /$ liter, aspartate aminotransferase $438 \mathrm{IU} /$ liter ( $<40 \mathrm{IU} /$ liter), alanine aminotransferase $148 \mathrm{IU} /$ liter ( $<40 \mathrm{IU} /$ liter $)$. The urinary excretion of dicaroxylic acids (adipic, suberic, sebacic, 3-hydroxy-sebacic, dodecenedioic, decadienedioic, decenedioic, 3-hydroxy-dodecenedioic acids) was slightly elevated during this episode. This mild dicarboxylic aciduria is not a common feature of CPT I deficiency, although it has been reported twice before $(6,8)$.

Since the child was diagnosed, she had several episodes of hypoglycemia and (mild) metabolic acidosis, which occurred during acute viral infection and/or because of feeding problems, necessitating administration of intravenous glucose and bicarbonate.

At $18 \mathrm{mo}$ of age she started to walk. Now at $30 \mathrm{mo}$ she is slightly hypotonic and falls frequently. Before her diagnosis she had several encephalopathic episodes lasting for 5 to $10 \mathrm{~d}$, which may have been caused by hypoglycemia due to the CPT I deficiency. The hypotonicity as well as the motor retardation are probably caused by these hypoglycemic episodes.

Cell culture conditions. Cell culture conditions were exactly as described previously (9).

Fatty acid $\beta$-oxidation. Fatty acid $\beta$-oxidation of $\left[9,10-{ }^{3} \mathrm{H}\right]-$ myristate and palmitate in fibroblasts was performed essentially as described by Manning and co-workers (10). To this end fibroblasts were harvested with trypsin-EDTA according to standard procedures and suspended in Ham's F10 medium. The cell density of the fibroblast suspension was estimated by light scattering $\left(\mathrm{OD}_{600}=0.14\right.$ corresponding to $\sim 0.05 \mathrm{mg} / \mathrm{ml}$ protein). From this suspension, $0.4 \mathrm{ml}(20$ $\mu \mathrm{g}$ protein) was seeded in $1-\mathrm{cm}^{2}$ glass vials and the cells were allowed to attach overnight. After washing the cells with PBS, $200 \mu$ of RPMI medium (without glucose) was added containing $20 \mathrm{mM}$ Hepes, $\mathrm{pH}$ 7.4, $100 \mu \mathrm{M}$ fatty acid (myristic or palmitic acid), 200,000 dpm tritium labeled fatty acid $\left(\left[9,10-{ }^{3} \mathrm{H}\right]\right.$-myristic or $\left[9,10-{ }^{3} \mathrm{H}\right]$-palmitic acid) obtained from Amersham (Arlington Heights, IL), and $1 \mathrm{mg} / \mathrm{ml}$ BSA (essentially fatty acid free) (Sigma Chemical Co., St. Louis, MO). The reaction was stopped by rapid cooling on ice-water after $2 \mathrm{~h}$ of incubation at $37^{\circ} \mathrm{C}$ and the labeled product $\left(\left[{ }^{3} \mathrm{H}\right]\right.$-water $)$ was measured as described (11). The remaining cells were homogenized in Triton/PBS ( $1 \mathrm{~g} /$ liter) by sonication and protein content was measured using the BCA kit from Sigma Chemical Co. (12).

Enzyme assays. Enzyme assays were performed as described before (13).

CPT activity. CPT activity was measured essentially as described by Demaugre and co-workers (5) with some modifications. The assay mixture (500 $\mu$ l final volume) contained $50 \mathrm{mM}$ Hepes, $\mathrm{pH} 7.4,150$ $\mathrm{mM} \mathrm{KCl}, 1 \mathrm{mM}$ EDTA, $1 \mathrm{mM}$ DTT, $1.3 \mathrm{mg} / \mathrm{ml} \mathrm{BSA}$ (essentially fatty acid free), $0.5 \mathrm{mM}$ L-carnitine (Sigma Chemical Co.), and 200,000 dpm L-[methyl- $\left.{ }^{14} \mathrm{C}\right]$-carnitine (New England Nuclear Life Science, Boston, MA), $0.1 \mathrm{mM}$ palmitoyl-CoA (Boehringer Mannheim, Mannheim, Germany). Reactions were started by adding fibroblasts (300 $\mu \mathrm{g}$ protein) or yeast homogenate (10-20 $\mu \mathrm{g}$ protein) and incubated for $10 \mathrm{~min}$ at $37^{\circ} \mathrm{C}$ with (CPT II activity) or without (CPT I + CPT II activity) $0.2 \mathrm{mM}$ malonyl-CoA, which is a specific inhibitor of CPT I but not of CPT II. Reactions were terminated by addition of $0.5 \mathrm{ml}$ $0.5 \mathrm{M} \mathrm{HCl}$ and the labeled product palmitoyl-carnitine was extracted with $n$-butanol as described (14).

Immunoblot analysis. Fibroblast homogenates were subjected to SDS-PAGE on a $7.5 \%$ acrylamide gel. CPT IA protein was visualized using a polyclonal antibody raised against rat CPT IA (gift from Prof. V.A. Zammit, Hannah Research Institute, Ayr, Scotland).

RNA isolation and cDNA synthesis. Total RNA was isolated from cultured skin fibroblasts using the acid guanidinium thiocyanate-phenol-chloroform extraction procedure (15) and was used to prepare cDNA (16).

Sequence analysis. The complete cDNA for CPT IA was amplified in three overlapping fragments using the following M13-tagged primers: fragment A: $-28 \mathrm{CPT} 1 \mathrm{f}-21 \mathrm{M} 135^{\prime}$-tgt aaa acg acg gcc agt CTC CGT AGC TGA CTC GGT AC-3' and 760CPT1rM13rev 5'cag gaa aca gct atg acc AGT TGC TGT TCA CCA TGA GC-3'; fragment B: 682CPT1f-21M13 $5^{\prime}$-tgt aaa acg acg gcc agt GCT ACA AAT
TAC GTG AGC GAC-3' and 1596CPT1rM13rev 5'-cag gaa aca gct atg acc GGT CTC TAT AAC CTC TTG AC-3'; fragment C: 1460CPT1f-21M13 5' -tgt aaa acg acg gcc agt ACG TCA TGT CCA TTG ACA GC-3' and 2351CPT1rM13rev 5' -cag gaa aca gct atg acc CGT TTT CCT TCC CAG CAG CTC-3'.

Sequence analysis of these PCR fragments using fluorescent labeled M13 primers (Amersham) was performed on an Applied Biosystems 377A automated DNA sequencer following the manufacturer's protocols.

Construction of expression plasmids. Two expression plasmids were constructed for expression of CPTI-454D (wild-type) and CPTI454G (mutant) in Saccharomyces cerevisiae.

The complete open reading frame of the gene coding for CPT IA was amplified in a PCR reaction using the following primer set: sense 5' -aaa aag ctt aaa ATG GCA GAA GCT CAC CAA GC-3' and anti-

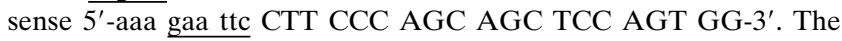
PCR products amplified from control and patient's cDNA were first inserted into pGEM-T (Promega Corp., Madison, WI). The cloned PCR products were sequenced to assess the integrity of the PCR process.

Plasmid pYES2 (Invitrogen Corp., Carlsbad, CA) was used to create $\mathrm{pYCPTI}-454 \mathrm{D}$ and $\mathrm{pYCPTI}-454 \mathrm{G}$ by cloning the ORF of control and mutant CPT IA, respectively, into the HindIII and EcoRI sites downstream of the GAL1 promoter.

Yeast culture and expression. Wild-type $S$. cerevisiae (INVSC2: MAT $\alpha$, his3-D200, ura3-167 obtained from Invitrogen) and the transformed strains were grown to $\mathrm{OD}_{600}=0.2$ in a medium composed of $0.67 \mathrm{~g} /$ liter yeast nitrogen base without amino acids (Difco Laboratories Inc., Detroit, MI) and $20 \mathrm{~g} /$ liter lactate, supplemented with the appropriate amino acids. Overnight induction was initiated by the addition of galactose to a final concentration of $2 \mathrm{~g} /$ liter. The cells were harvested and spheroplasts were prepared according to standard procedures (17). Spheroplasts were homogenized by sonication in a buffer containing $10 \mathrm{mM}$ Mops, $\mathrm{pH}$ 7.4, $160 \mathrm{mM} \mathrm{KCl,} 1 \mathrm{mM}$ EDTA, and $1 \mathrm{mM}$ PMSF. The homogenates were centrifuged at 10,000 $\mathrm{g}$ for $30 \mathrm{~s}$ and the supernatants were used for enzyme activity measurements.

\section{Results}

Fatty acid oxidation and enzyme analysis. The organic acid profile in urine of the index patient suggested a fatty acid oxidation defect. This was confirmed by initial $\beta$-oxidation measurements in lymphocytes. Biochemical studies in fibroblasts from the index patient showed that the oxidation of the two longchain fatty acids myristate and palmitate was markedly decreased (Table I). To exclude that this was due to a defect in either very-long chain acyl-CoA dehydrogenase or mito-

Table I. Fatty Acid $\beta$-oxidation, CPT Activity $( \pm S D)$ and Immunoblot Analysis Using Antibodies Raised against Rat CPT IA in Fibroblasts from Control Subjects and the Patient

\begin{tabular}{ccc}
\hline Parameter measured & Patient & Controls \\
\hline Overall $\beta$-oxidation activity & & \\
Myristic acid $(\mathrm{nmol} / \mathrm{h} / \mathrm{mg})$ & 1.4 & $5.9 \pm 2.4(n=91)$ \\
Palmitic acid $(\mathrm{nmol} / \mathrm{h} / \mathrm{mg})$ & 0.5 & $7.8 \pm 3.3(n=119)$ \\
Enzyme activity measured & & \\
CPT I (nmol/min/mg) & $<0.01$ & $0.58 \pm 0.24(n=17)$ \\
CPT II (nmol/min/mg) & 0.70 & $0.61 \pm 0.23(n=17)$ \\
Immunoblot analysis & & \\
88-kD cross-reactive & Virtually absent & Present \\
material & $(n=2)$ & $(n=2)$ \\
& & \\
\hline
\end{tabular}




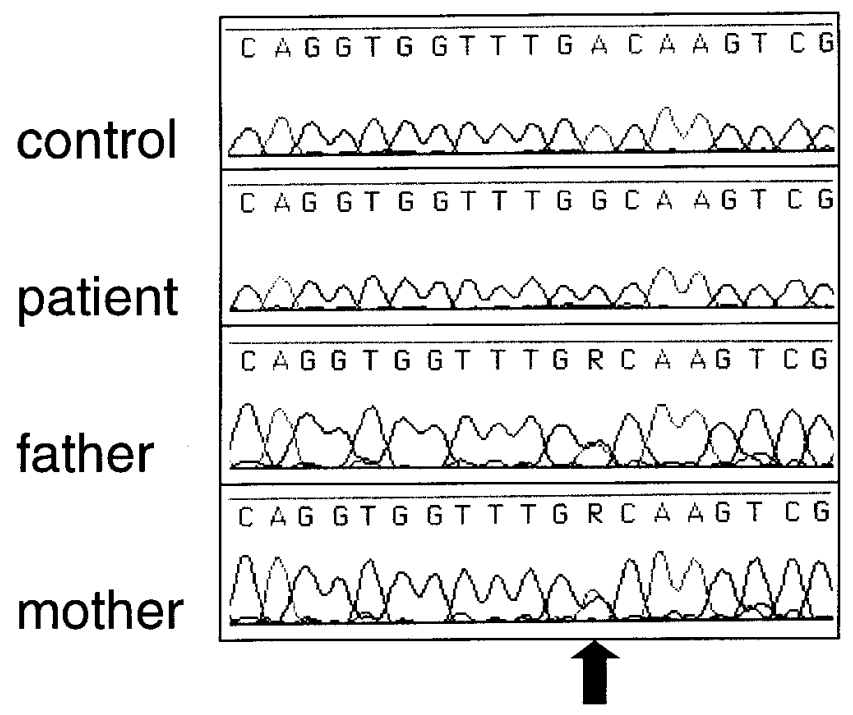

Figure 1. Electropherogram of a control subject, the index patient, and both parents showing the sequence surrounding the A1361G mutation (indicated by an arrow) in CPT IA.

chondrial trifunctional protein, two key enzymes in the mitochondrial long-chain fatty acid oxidation, we determined the activity of acyl-CoA dehydrogenase, enoyl-CoA hydratase, 3-hydroxyacyl-CoA dehydrogenase, and thiolase and found them to be normal (not shown). This suggested that the defect could be at the level of the carnitine-dependent transport shuttle. Of the three enzymes involved in this shuttle we found normal activities for CPT II and CAC (not shown), whereas CPT I was found to be fully deficient (Table I). Earlier studies have shown that fibroblasts express CPT IA but not CPT IB (7) which led us to study CPT IA in more detail.

Immunoblot analysis. To study CPT IA at the protein level, we first performed immunoblot experiments using an antibody raised against CPT IA. In control fibroblasts the CPT IA protein was found to be clearly present, whereas the signal was very weak in the patient's fibroblasts (Table I).

Sequence analysis. The cDNA sequence encoding human CPT IA has been reported (7). To determine the genetic defect in our patient we sequenced the coding region for CPT IA using cDNA obtained from fibroblasts of control subjects and the index patient.

In two controls we found two differences when compared with the reported sequence: an $\mathrm{A} 1435 \mathrm{C}$ and an $\mathrm{A} 1702 \mathrm{G}$ changing the glutamine at position 479 to a proline and the threonine at position 568 into an alanine, respectively. Since these fibroblasts were found to contain normal CPT I activity, these differences likely represent polymorphisms.

Analysis of the patient's cDNA revealed the A1435C polymorphism, a silent G963A mutation, and an A1361G missense mutation (Fig. 1). As a consequence of this mutation the aspartate at position 454 is changed into a glycine (D454G). The finding of the A1361G mutation in a heterozygous form in both parents (Fig. 1) indicates that the patient is truly homozygous for this mutation.

Expression studies. The aspartate at position 454 is located in a region which is highly conserved among 17 reported carnitine acyltransferases (Fig. 2). To investigate the effect of the mutation on the enzyme activity we expressed in the yeast $S$. cerevisiae both the wild-type and the mutant CPT I. S. cerevisiae is not only easy to manipulate genetically but is particularly suited for this study because yeast lacks endogenous CPT activity. Homogenates of transformed cells (derived from four to six independent transformants) as well as untransformed cells were prepared and CPT activity was measured (Fig. 3). In agreement with literature data, wild-type yeast and yeast transformed with the expression vector (pYES2) only did not display any CPT activity. On the other hand, cell homogenates from transformants with pYCPTI-454D (wild-type CPT IA) were found to contain high CPT IA activity. The specific activity was $\sim 10$-fold higher than observed in fibroblasts. In contrast, transformants with pYCPTI-454G (mutant CPT IA) displayed only $2 \%$ of the expressed wild-type CPT IA. These data show that the D454G substitution causes loss of CPT IA activity and is responsible for the hepatic CPT I deficiency in this patient.

\section{Discussion}

So far 11 patients with CPT I deficiency have been reported in literature. The patient described in this report showed many of the clinical abnormalities described in other patients with CPT I deficiency including hepatomegaly and periods of muscle weakness and fainting due to hypoglycemia. Our observation

CPT1A_human
CPT1A_rat
CPT1B_human
CPT1B_rat
CPT2_human
CPT2_rat
COT_cow
COT_rat
CAT_human
CAT_mouse
CAT_pigeon
CAT_C.tropicalis
CAT_S.cerevisiae
CAT_E.nidulans
ChAT_human
ChAT_rat
ChAT_D.melanogaster

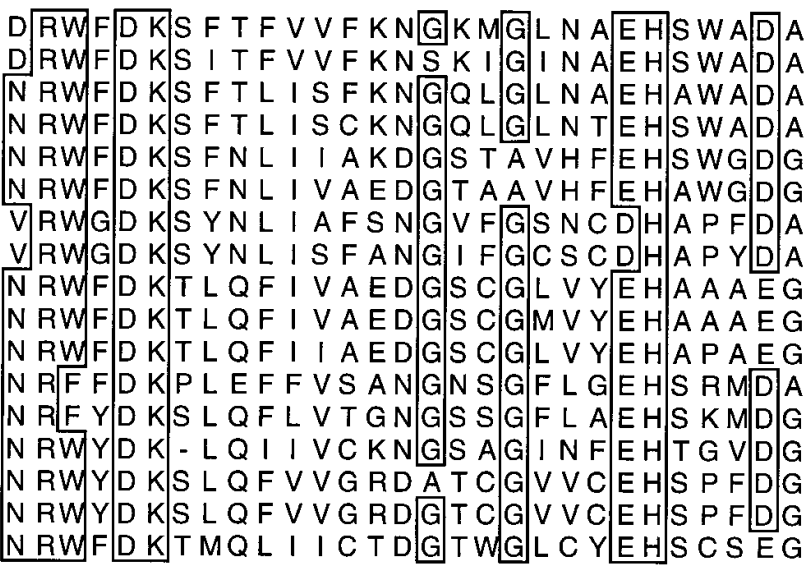

Figure 2. Comparison of different acyltransferases from different species: CPT IA from human and rat; CPT IB from human and rat; CPT II from human and rat; carnitine octanoyltransferase (COT) from bovine and rat; carnitine acetyltransferase $(C A T)$ from human, mouse, pigeon, Candida tropicalis, S. cerevisiae, and Emericella nidulans; choline acetyltransferase (ChAT) from human, rat, and Drosophila melanogaster. The consensus pattern is: [NDV]$\mathrm{R}-[\mathrm{WF}]-\mathrm{X}-\mathrm{D}-\mathrm{K}-\mathrm{X}(0,1)-[\mathrm{LIVMFY}]-\mathrm{X}-$ [LIVMFY](2)-X(3)-[DN]-[GSA]-X(2)$[\mathrm{GA}]-\mathrm{X}(3)-[\mathrm{ED}]-[\mathbf{H}]-\mathrm{X}(3)-[\mathrm{DE}]-[\mathrm{AG}]$ with the active histidine residue in bold. 


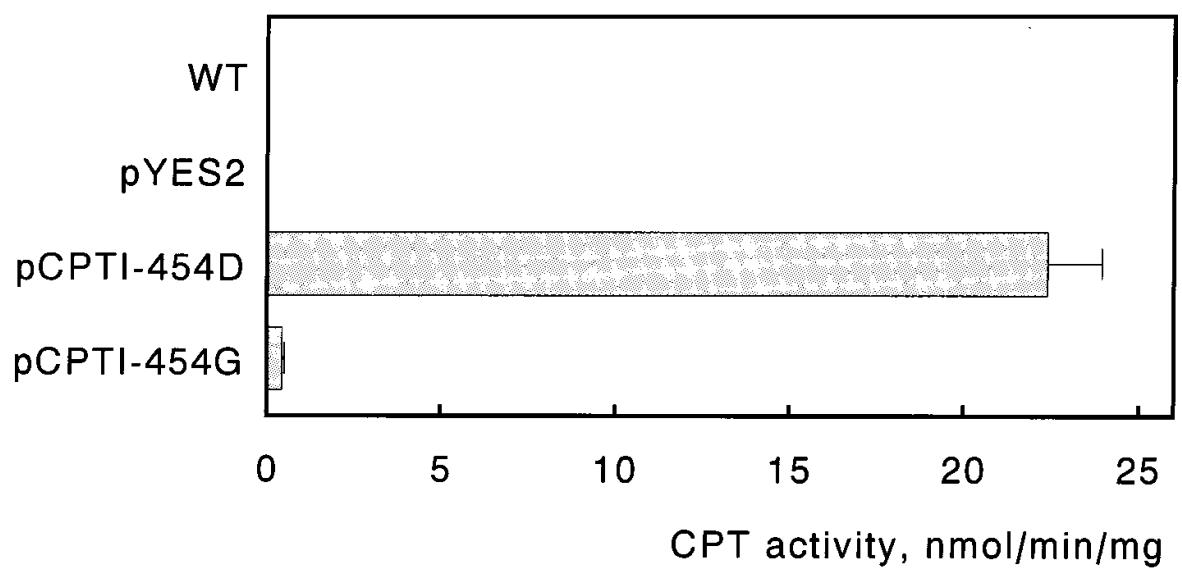

Figure 3. Expression of human CPT IA in S. cerevisiae. Wild-type yeast (WT) cells were transformed with different expression plasmids containing the open reading frame of the wild-type CPT IA (pYCPTI454D), mutant CPT IA (pYCPTI-454G), and the expression vector (pYES2) only. CPT activity was measured in homogenates prepared from spheroplasts as described in Methods. that fibroblasts of this patient were deficient in CPT I activity indicated that the patient is deficient in CPT IA, which is the only CPT I isoform expressed in fibroblasts (7). Molecular analysis of CPT IA cDNA of this patient revealed a homozygous A1361G missense mutation changing the aspartate at position 454 into a glycine. This mutation was associated with a dramatic decrease in CPT activity as demonstrated by expression studies in $S$. cerevisiae: mutant CPT IA (454G) displayed only $2 \%$ of the activity of expressed wild-type CPT IA (454D). These data show that the $\mathrm{D} 454 \mathrm{G}$ mutation is the disease-causing mutation.

Alignment of different reported carnitine acyltransferases reveals structural sequences shared within this transferase family. The histidine at position 473 is thought to be the active residue (18-20) and is located in a well-conserved region (see Fig. 2). The aspartate at position 454 which is mutated in our patient is located 19 amino acids upstream of this histidine and is conserved among all transferases indicative for an important role. Indeed, the role of an aspartate as part of a "catalytic triad" has been suggested by Brown et al. (20). To test this hypothesis, they performed site-directed mutagenesis of CPT II and changed the aspartate at position 353 (homologous to the aspartate at position 454 in CPT IA) into an alanine. When expressed in yeast, the resulting mutant CPT II was unstable as judged by immunoblots and the lysate contained $<1 \%$ of the CPT activity of yeast-expressed wild-type CPT II. They concluded that the majority of the synthesized protein became misfolded and subsequently degraded and that only a small portion of the translated product achieved the correct conformation having normal activity.

The finding that CPT IA protein was hardly detectable in the patient's fibroblasts upon immunoblot analysis suggests that the D454G mutation also renders the CPT IA protein unstable, thus explaining the deficient CPT I activity in our patient.

\section{Acknowledgments}

We are grateful to Prof. Zammit who kindly provided the CPT IA antibodies.

This work was supported in part by the Elias Fund/Technion VPR fund (grant 181-421).

\section{References}

1. McGarry, D.J., and N.F. Brown. 1997. The mitochondrial carnitine palmitoyltransferase system. Eur. J. Biochem. 244:1-14.

2. Britton, C.H., D.W. Mackey, V. Esser, D.W. Foster, D.K. Burns, D.P. Yarnall, P. Froguel, and J.D. McGarry. 1997. Fine chromosome mapping of the genes for human liver and muscle carnitine palmitoyltransferase I (CPT1A and CPT1B). Genomics. 40:209-211.

3. Di Mauro, S., and P.M.M. Di Mauro. 1973. Muscle carnitine palmitoyl transferase deficiency and myogloburia. Science. 182:929-931.

4. Brounes, P.F., J.M. Saudubray, C. Marsac, M. Odievre, and J. Girard 1981. Fasting hypoglycaemia resulting from hepatic carnitine palmitoyltransferase deficiency. J. Pediatr. 98:742-746.

5. Demaugre, F., J.P. Bonnefont, M. Colonna, C. Cepanec, J.P. Leroux, and J.M. Saudubray. 1991. Infantile form of carnitine palmitoyltransferase II deficiency with hepatomuscular symptoms and sudden death. Physiopathological approach to carnitine palmitoyltransferase II deficiencies. J. Clin. Invest. 87: 859-864.

6. Bergman, A.J.I.W., R.A.M.G. Donckerwolcke, M. Duran, J.A.M Smeitink, B. Mousson, C. Vianey-Saban, and B.T. Poll-The. 1994. Rate-dependent distal renal tubular acidosis and carnitine palmitoyltransferase I deficiency. Pediatr. Res. 36:582-588.

7. Britton, C., R.A. Schultz, B. Zhang, V. Esser, D.W. Foster, and D.J. McGarry. 1995. Human liver mitochondrial carnitine palmitoyltransferase I. Characterization of its cDNA and chromosomal localization and partial analysis of the gene. Proc. Natl. Acad. Sci. USA. 92:1984-1988.

8. Falik-Borenstein, Z.C., S.C. Jordan, J.M. Saudubray, M. Brivet, F. Demaugre, J. Edmond, and S.D. Cederbaum. 1992. Brief report: renal tubular acidosis in carnitine palmitoyltransferase type I deficiency. N. Engl. J. Med. 327: 24-27.

9. Wanders, R.J.A., C.W.T. van Roermund, M.J.A. van Wijland, R.B.H. Schutgens, J. Heikoop, H. van der Bosch, A.W. Schram, and J.M. Tager. 1987 Peroxisomal fatty acid beta-oxidation in relation to the accumulation of very long chain fatty acids in cultured skin fibroblasts from patients with Zellweger syndrome and other peroxisomal disorders. J. Clin. Invest. 80:1778-1783.

10. Manning, N.J., S.E. Olpin, R.J. Pollitt, and J. Webley. 1990. A comparison of $\left[9,10-{ }^{3} \mathrm{H}\right]$ myristic acids for the detection of defects of fatty acid oxidation in intact cultured fibroblasts. J. Inher. Metab. Dis. 13:58-68.

11. Moon, A., and W.J. Rhead. 1987. Complementation of fatty acid oxidation disorders. J. Clin. Invest. 79:59-64.

12. Smith, P.K., R.I. Krohn, G.T. Hermanson, A.K. Mallia, F.H. Gartner, M.D. Provenzano, E.K. Fujimoto, N.M. Goeke, B.J. Olson, and D.C. Klenk. 1985. Measurement of protein using bicinchroninic acid. Anal. Biochem. 150: 76-85.

13. Wanders, R.J.A., L. IJlst, F. Poggi, J.P. Bonnefont, A. Munnich, M Brivet, D. Rabier, and J.M. Saudubray. 1992. Human trifunctional protein deficiency: a new disorder of mitochondrial fatty acid oxidation. Biochem. Biophys. Res. Commun. 188:1139-1145.

14. McGarry, J.D., G.F. Leatherman, and D.W. Foster. 1977. Carnitine palmitoyltransferase I. The site of inhibition of hepatic fatty acid oxidation by malonyl-CoA. J. Biol. Chem. 253:4128-4136.

15. Chomczynski, P., and N. Sacchi. 1987. Single-step method of RNA isolation by acid guanidinium thiocyanate-phenol-chloroform extraction. Anal. Biochem. 162:156-159.

16. IJlst, L., R.J.A. Wanders, S. Ushikubo, T. Kamijo, and T. Hashimoto. 
1994. Molecular basis of long-chain 3-hydroxyacyl-CoA dehydrogenase deficiency: identification of the major disease-causing mutation in the $\alpha$-subunit of the mitochondrial trifunctional protein. Biochim. Biophys. Acta. 1215:347-350.

17. Yaffe, M.P. 1991. Mitochondrial function and assembly. In Guide to Yeast Genetics and Molecular Biology. Methods in Enzymology. Vol. 194. C. Guthrie and G.R. Fink, editors. Academic Press, San Diego, CA. 627-643.

18. Chase, J.F.A., and P.K. Tubbs. 1970. Specific alkylation of a histidine residue in carnitine acetyltransferase bromoacetyl-L-carnitine. Biochem. J. 116:
713-720.

19. Schmalix, W., and W. Bandlow. 1993. The ethanol-inducible YAT1 gene from yeast encodes a presumptive mitochondrial outer carnitine acetyltransferase. J. Biol. Chem. 268:27428-27439.

20. Brown, N.F., R.C. Anderson, S.L. Caplan, D.W. Foster, and J.D. McGarry. 1994. Catalytically important domains of rat carnitine palmitoyltransferase II as determined by site-directed mutagenesis and chemical modification. J. Biol. Chem. 269:19157-19162. 